\title{
Hemorragia digestiva por fístula de artéria subclávia direita anômala com o esôfago
}

\author{
R. Colleoni Neto, A. Figueira, E. Belassai, M.E. J orge J R., J .C. Del Grande, S.H. Cardoso*, C.M. Haddad \\ Disciplina deGastroenterol ogia Cirúrgica do Departamento deCirurgia e*Disciplina dePatologia M édica doDepartamento deAnatomia \\ Patológica. Universidade Federal de São Paulo - Escola Paulista de Medicina, São Paulo, SP.
}

\begin{abstract}
RESUMO - As hemorragias decorrentes das comunicações arteriais com o esôfago são raras e letais em praticamente todos os casos. 0 tratamento cirúrgico imediato é a única opção terapêutica curativa. Os autores apresentam um caso de hemorragia por fístula de artéria subclávia direita anômala com o esôfago, no qual o diagnóstico foi esclarecido apenas na necropsia, após três operações. E sta alteração anatômica é encontrada em $0,5 \%$ da população geral. Raramente se estabelece comunicação desta artéria com o esô-
\end{abstract}

\section{INTRODUÇÃO}

As hemorragias decorrentes das comunicações arteriais com o tubo digestivo, particularmente com o esôfago, são raras e geralmente fatais, não só por serem caracteristicamente intensas, mas também por dificuldades em determinar sua origem e indicar o tratamento cirúrgico em momento oportuno ${ }^{1}$.

\section{APRESE NTAÇÃO DO CASO}

S.T.M., 15 anos, masculino, portador de distrofia muscular progressiva, foi admitido no Pronto Socorro do Hospital São Paulo apresentando hematêmese havia quatro horas. Há cinco dias, vinha fazendo uso de áci do acetil sal i ćl ico e sulfametoxazol-trimetoprima por causa de quadro infeccioso das vias aéreas superiores. Ao exame físico, apresentava intensa palidez das mucosas, pressão arterial de $75 \times 55 \mathrm{mmH}$ g e pul so com 120 batimentos por minuto. Destacavam-se, ao exame físico: cifoescoliose acentuada, atrofia muscular, membros fletidos e anquilosados e presença de melena ao toque retal. A dosagem inicial de hemogl obina era $6,6 \mathrm{~g} / \mathrm{dL}$ e o hematócrito, $27 \%$. Após reposição de duas unidades de concentrado de hemácias, foi submetido à endoscopia, que não identificou alterações no esôfago e no estômago, mas demonstrou uma úlcera na parede posterior do bulbo duodenal, com um vaso visível e parcialmente recoberta por coágulo. Procedeu-se a he- fago, como conseqüência de dilatação aneurismática ou de traumatismo provocado pela permanência prolongada de sonda nasogástrica, como, provavel mente, ocorreu com esse doente. $O$ tratamento cirúrgico dependerá do reconhecimento precoce dos sinais diagnósticos sugestivos de comunicação arterial com o esôfago antes que a hemorragia traga repercussão sistêmica.

UNITERMOS: Fístula do esôfago. Hemorragia digestiva. Endoscopia.

mostasia endoscópica pela injeção de álcool absoluto. Foi instituído tratamento clínico com administração de ranitidina e antiácidos, e uma sonda nasogástrica foi introduzida. Após dois dias, apresentou outro epi sódio de hemorragia digestiva com grave repercussão hemodinâmica, sendo indicado tratamento cirúrgico, que demonstrou presença de uma úlcera duodenal sem sangramento ativo. Foi realizada vagotomia troncular e antrectomia com gastroduodenostomia. Evoluiu bem até o quarto dia de pós-operatório, quando apresentou hematêmese com sangue vivo em grande quantidade, acompanhada de choque hipovol êmico. Submetido a laparotomia de urgência, identificou-se grande quantidade de sangue e coágulos no estômago. A provável origem do sangramento foi local izada na região da transi ção entre a gastrorrafia da curvatura menor e a anastomose, onde foram aplicados pontos hemostáticos. Após estabilização hemodinâmica, o doente foi transferido para a unidade de terapia intensiva, quando poucas horas depois apresentou sangramento abundante pela sonda nasogástrica com choque hipovol êmico. Nova laparotomia de urgência evidenciou que o sangramento se originava no esôfago. Foi realizado tamponamento esofágico com sonda de SengstakenBlakemore, interrompendo-se a hemorragia. A tentativa de realização de endoscopia intra-operatória não teve sucesso, pois, quando o balão da sonda de tamponamento era desinsuflado, ocorria hipotensão grave. A pós transfusão sanguínea 


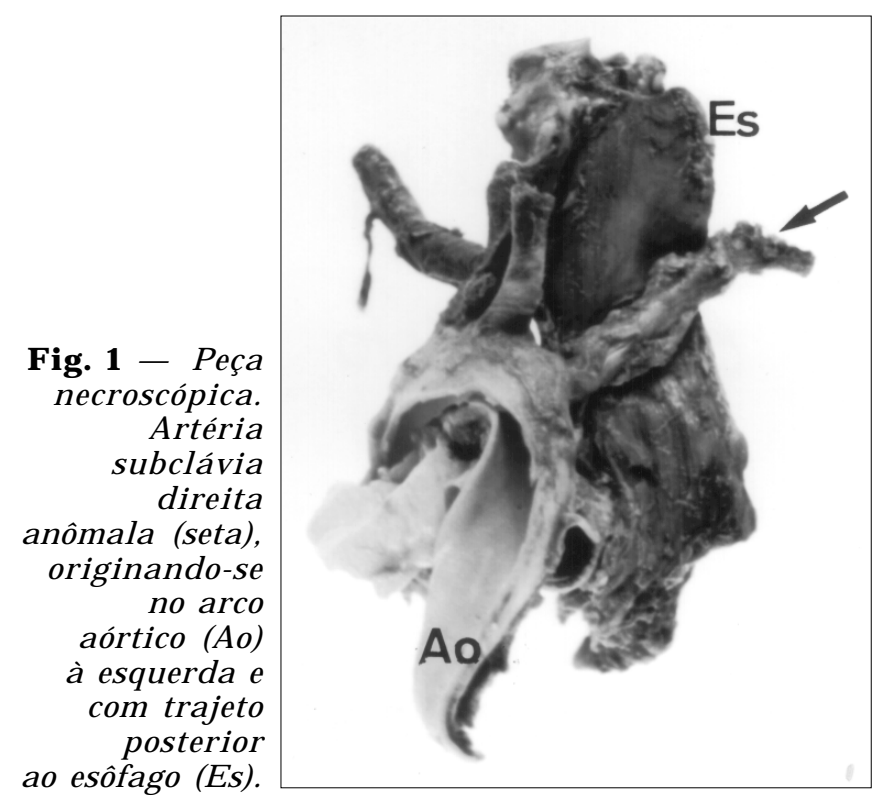

maciça e reversão de duas paradas cardiorrespiratórias, fal eceu poucas horas após a reoperação.

O exame necroscópico revel ou a existência de artéria subclávia direita anômala (ASDA), originada na aorta descendente e dirigindo-se para a direita, cruzando, posteriormente, o esôfago (fig. 1). I dentificou-se uma fístula entre a parede posteri or do esôfago e a parede anterior da artéria aberrante (fig. 2), responsável pela hemorragia maciça e fatal.

\section{DISCUSSÃO}

Artéria subclávia direita anômala (ASDA) é a malformação mais comum do arco aórtico, podendo ocorrer de forma quase sempre assintomática em $0,5 \%$ da população. Deve-se ao desaparecimento do quarto arco aórtico, do qual se origina a porção proximal da artéria subclávia direita. Dessa forma, a ASDA emerge da aorta descendente, cruzando obliquamente o mediastino posterior, da esquerda para a direita, entre o esôfago e a coluna vertebral. É considerada, portanto, um anel vascular incompleto. Sua manifestação clínica mais freqüente é conseqüência da compressão do esôfago, o que ocorre, geralmente, em crianças, manifestando-se pela denominada disfagia Iusória. Mais raramente, há compressão traqueal determinando sintomas respiratórios e também podem existir sintomas relacionados a cardiopatias congênitas associadas.

A ocorrência de hemorragia por comunicação de ASDA com o esôfago é situação extremamente rara, sendo descrita, basicamente, em duas situações. A primeira corresponde à dilatação aneurismática

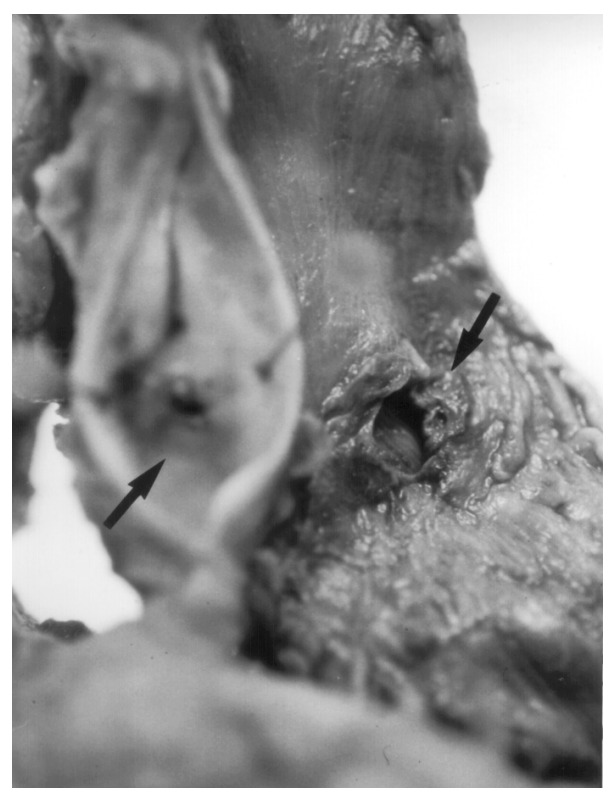

Fig. 2 - Peça
necroscópica.
Presença de
orifícios
fistulosos
situados na
parede
posterior do
esôfago (seta à
direita) e
na parede
anterior da
artéria
subclávia
direita
anômala (seta
à esquerda).

na origem dessa artéria, lesão rara, conhecida como "divertículo de Kommerell", geral mente assintomática e observada em adultos e idosos ${ }^{4-6}$. Essa lesão provocaria erosão progressiva da parede esofagiana, à semelhança do que ocorre com maior freqüência entre um aneurisma arteriosclerótico da aorta abdominal e o duodeno ${ }^{7}$. A outra situação corresponde à perfuração da lesão determinada pelo atrito prolongado de sonda nasogástrica ${ }^{2,7,8}$, como, provavel mente, ocorreu nesse caso. O diagnóstico da ASDA poderá ser feito por meio de esofagograma pela demonstração de compressão da parede esofágica posterior, ou, ainda, pelo exame endoscópico ao identificar uma compressão pulsátil na parede posterior do esôfago. Estabelecido o diagnóstico, a conduta proposta é a dissecção do vaso, em seu trajeto retroesofágico, com secção e ligadura dele em suas extremidades proximal e distal ${ }^{3,9}$. Na maioria das vezes, esse procedimento não prejudica a perfusão do membro superior direito, que, nessas condições, será mantida por circulação colateral. Pode-se, ainda, para evitar isquemia do membro, praticar anastomose do segmento distal da ASDA com a carótida direita ${ }^{9}$. A via de acesso preferida é a toracotomia esquerda.

O sangramento causado por comunicação arterial com o esôfago manifesta-se tipicamente por hemorragia sentinela, que corresponde a hematêmese volumosa de sangue arterial seguida, freqüentemente, de um período assintomático, com duração variável, até a ocorrência de um novo epi sódio de sangramento súbito e geralmente fatal 1,8. A penas o reconhecimento desta situação permitirá o estabelecimento de conduta cirúrgica imediata, o que não ocorre na grande maioria dos 
casos $^{2}$. Este reconhecimento torna-se ainda mais difícil quando a hemorragia é secundária à complicação de uma anomalia congênita sem outras repercussões clínicas, como nesse caso. O sangramento maciço impede um exame endoscópico conclusivo. O diagnóstico da origem da hemorragia no esôfago, muitas vezes, é realizado no intra-operatório de uma laparotomia exploradora ${ }^{5}$. O tamponamento com balão de Sengstaken-Blakemore pode ser útil no controle temporário do sangramento ${ }^{1}$, como pudemos observar nesse caso. A técnica operatória de escol ha consiste na secção e ligadura do vaso anômalo e desbridamento e sutura do orifício fistuloso do esôfago. Podem ser realizadas, associadamente, esofagostomia cervical, gastrostomia e drenagem pleural. Quando a situação clínica do doente não permitir abordagem direta da fístula, poder-se-á realizar apenas exclusão esofágica, deixando para um segundo tempo a correção da anomalia².

Devemos destacar a extrema dificuldade diagnóstica observada nos portadores de hemorragia secundária às comunicações arteriais com o esôfago, particularmente nas anomalias vasculares, determinando alta mortalidade $\mathrm{e}^{4,6-8}$. Somente o reconhecimento de seus sinais permite o estabelecimento da conduta cirúrgica em momento oportuno, ou seja, antes que o sangramento intenso e repetido determine repercussões sistêmicas. O diagnóstico poderá ser auxiliado pela suspeita ou conhecimento prévio da existência de uma alteração vascular.

\section{SUMMARY}

\section{Upper gastrointestinal bleeding from a fistula of a right anomalous subclavian artery to the esophagus}

Bleeding from an arterio-esophageal fistula is a rare and nearly lethal condition and surgical treatment is the only curative option. We report a case of bleeding from a fistula from an aberrant right subclavian artery to the esophagus. Diagnosis was made only at necropsy, despite of three previous laparotomies. This anatomical variation is found in $0.5 \%$ of the general population. Development of a communication between this artery and the esophagus, secondary to aneurysmatic dilatation or to prolonged nasogastric intubation, as probably occurred with this patient, is a extremely rare condition. Surgical treatment depends on the early recognition of clinical signs of the arterio-esophageal comunication, before the onset of systemic complications of hypovolemic shock. [Rev Ass Med Brasil 1998; 44(2): 149-51.]

KEY WORDS: Esophageal fistula. Gastrointestinal bleeding. Endoscopy.

\section{REFERÊNCIAS BIBLIOGRÁFICAS}

1. Rasslan S, Fava J, Mandia NetoJ et al. Hemorragia fatal por comunicação arterial com o trato digestivo. Rev Ass Med Brasil 1981; 27: 131-4.

2. Guzzetta PC, Newman K, Ceithaml E. Succesful management of aberrant subclavian arterio-esophageal fistula in an infant. Ann Thorac Surg 1989; 47: 308-9.

3. Macksoud Filho J G, Gonçalves MEP, Tannuri U, Macksoud J G. Compressões do esôfago e da traquéia por anomalias do arco aórtico na infância. Rev Ass Med Brasil 1993; 39: 165-9.

4. Glock Y, Chaffai M, Tasrini J et al. Hematemese par fissuration oesophagienned'un aneurysmesur arteria lusoria. Ann Chir 1984; 38: 541-7.

5. Lynn RB. Kommerell's diverticulum with esophago-arterial fistula. Can J Surg 1969; 12: 331-3.

6. Reynes J B, Erratia CA, Ercorreca FJ B, Landa AO. Aneurysm of right subclavian artery with esophageal perforation. Chest 1976; 70: 105.

7. Edwards BS, Edwards WD, Connolly DC, Edwards J E. Arterial-esophageal fistulae developing in patients with anomalies of the aortic arch system. Chest 1984; 86: 732-5.

8. Merchant FJ, Nichols RL, Bombeck CT. Unusual complication of nasogastric esophageal intubation: erosion into an aberrant right subclavian artery. J Cardi ovasc Surg 1977; 18: 147-50.

9. Hennessy TPJ . Miscellaneous. In Hennessy TPJ \& Cuschieri A (eds). Surgery of the oesophagus. London, BailliereTindall, 1986; 353-5. 